\title{
Disappearance and formation rates of microaneurysms in early diabetic retinopathy
}

Department of Ophthalmology, Helsinki University Central Hospital T Hellstedt

I Immonen

Correspondence to: Timo Hellstedt, MD, Department of

Ophthalmology, Helsinki University Hospital, University Hospital, 00290 Helsinki, Finland.

00290 Helsinki, Finland. 17 October 1995

T Hellstedt, I Immonen

\begin{abstract}
Aim-To analyse the formation and disappearance rates of individual microaneurysms in mild background retinopathy.

Methods-Three fluorescein angiograms were taken at 1 year intervals during a 2 year follow up from 24 type 1 diabetics with mild background retinopathy. Microaneurysms were identified and localised twice from each angiogram using a computerised system for retrieval of the coordinates for each microaneurysm. Microaneurysms identified similarly in both sessions were then processed further to obtain rates of microaneurysm formation and disappearance, and microaneurysm count changes. Results-In the whole material the total number of microaneurysms increased from 298 to 436 from baseline to 2 years. During the 2 year follow up 395 new microaneurysms formed and 258 microaneurysms disappeared. Of the microaneurysms present at baseline $174(58 \%)$ were still present at the 1 year and 142 $(48 \%)$ at the 2 year follow up. In patients with good glucose control $\left(\mathrm{HbA}_{\mathrm{lc}}\right.$ $\leqslant 7.5 \mathrm{mmol}$ ) microaneurysm formation showed a trend of being decreased whereas microaneurysm disappearance did not correlate with glucose control.

Conclusion-Background diabetic retinopathy is a dynamic process. A significant proportion of microaneurysms present disappear within 2 years. This is compensated for by formation of new microaneurysms, the resultant net changes in microaneurysm counts being small. Microaneurysm formation and disappearance rates are new variables of diabetic retinopathy and may prove to be more sensitive indicators of the progression patterns of background diabetic retinopathy than microaneurysm count changes.

(Br f Ophthalmol 1996; 80: 135-139)
\end{abstract}

Microaneurysms are the first clinically detectable sign of early diabetic retinopathy. The number of microaneurysms has been used to classify early diabetic retinopathy ${ }^{12}$ and the number of microaneurysms at baseline predicts further progression of diabetic retinopathy. ${ }^{3} 4$

Although some studies have reported the disappearance of these lesions, ${ }^{35}$ the fate of individual microaneurysms has not been studied much, in spite of their importance in the pathogenesis of diabetic retinopathy. We report here the fate of individual microaneurysms during a 2 year follow up in a group of diabetic patients with mild background retinopathy.

\section{Materials and methods}

We studied 43 eyes of 24 patients with insulin dependent diabetes. Five eyes were excluded because of the bad quality of the angiograms. The patients ranged in age from 23 to 69 years, the median age being 39 . The mean duration of diabetes was 18.3 (range 9 to 34 ) years. Of the patients, 10 were female and 14 male. All of the patients had mild background retinopathy and none of them developed proliferative or preproliferative retinopathy during follow up.

The patients gave their informed consent to the study, which was also approved by the institutional review committee of the Department of Ophthalmology, Helsinki University Hospital. The tenets of the Helsinki Declaration were followed.

Three fluorescein angiograms of each fundus were taken at 1 year intervals. The angiograms were taken with a Canon CF 60 camera, using Kodak T-Max 400 film with Exciter SE 40 and Barrier SB 50 filters after an intravenous injection of $12 \mathrm{mg} / \mathrm{kg}$ sodium fluorescein (Fluorescite, Alcon, Fort Worth, TX, USA).

In this study, we used central $60^{\circ}$ images centred on the fovea and taken at the late arterial phase (generally $12-15$ seconds after the injection). A rectangular area of $4 \times 6$ disc diameters extending from $1 \mathrm{DD}$ nasal of the optic disc to $5 \mathrm{DD}$ temporal of the disc was analysed. The criterion for classifying a lesion as a microaneurysm in the fluorescein angiograms was a distinctly round hyperfluorescent lesion $>20 \mu \mathrm{m}$ and $<125 \mu \mathrm{m}$ in diameter. Saccular capillary ends were considered to be microaneurysms.

The microaneurysms were localised and identified as described previously. ${ }^{6}$ In brief, the photographs were projected on a magnetic digitising table, and two anatomical points were selected as reference points. The lesions to be analysed were then marked using a mouse equipped with a crosshair ring and their coordinates in relation to the reference points were recorded and stored in a computer. We used a program that mathematically forms a small circle around each lesion. The diameter of this circle corresponded to $33 \mu \mathrm{m}$ at the retina. All coordinates within this circle were considered to represent the same lesion. This feature makes the program insensitive to small variations in 
localisation and photography such as small differences in angle of view or magnification. Generally, a map was drawn of some anatomical features and the microaneurysms present in the baseline fluorescein angiograms were drawn on a paper placed on the digitising table. With the aid of this map the lesions present in the previous angiogram could be sought for specially. In a subset of patients, the microaneurysms were also identified without premarking the lesions found in the first angiogram.

All angiograms were analysed twice by the same observer ( $\mathrm{TH}$, ophthalmologist with experience at evaluating diabetic fluorescein angiograms), with a 2 month interval, and no data from the previous analysis were consulted in the verification session. Thus, it was possible to exclude lesions that were not identified and similarly localised in both sessions. Unless stated otherwise, we used combined data from microaneurysms that were identified similarly in the two sessions. To evaluate the reproducibility of the identification/localisation procedure, we calculated an agreement rate between data from the two analyses of a fundus image. The agreement rate was calculated from the formula $(A /(A+B+C)) \times 100$, where $A$ is the number of microaneurysms detected/localised identically in both sessions, $B$ the total number of microaneurysms detected only in the first session, and $C$ the number of microaneurysms detected only at the second session. To test the intergrader variation, a subset of the angiograms was tested by another grader (II, vitreoretinal specialist) and the agreement rate between the observers was calculated. The differences in agreement were evaluated using the McNemar test, and the microaneurysm disappearance/formation rates between different patient groups using the Mann-Whitney test.

For comparison of the 2 year changes in microaneurysm formation, disappearance, and microaneurysm count between the different groups of patients, we calculated the following variables for each patient:

The microaneurysm formation rate index (baseline -2 years) $=$ (cumulative number of new microaneurysms detected at the 1 and 2 year follow ups/number of microaneurysms at baseline) $\times 100$.

The microaneurysm disappearance rate index (baseline -2 years) $=$ (cumulative number of microaneurysms disappearing at the 1 and 2 year follow ups/number of microaneurysms at baseline) $\times 100$.

The microaneurysm count change index $=$ (number of microaneurysms at 2 years / number of microaneurysms at baseline) $\times 100$.

The cumulative number of new microaneurysms during follow up=new lesions appearing between baseline and 1 year+new lesions appearing between 1 and 2 year follow ups.

These variables were calculated either from the mean values of both eyes or from the values of the only eye with data available. In the calculation of the microaneurysm formation and disappearance rate indexes, the

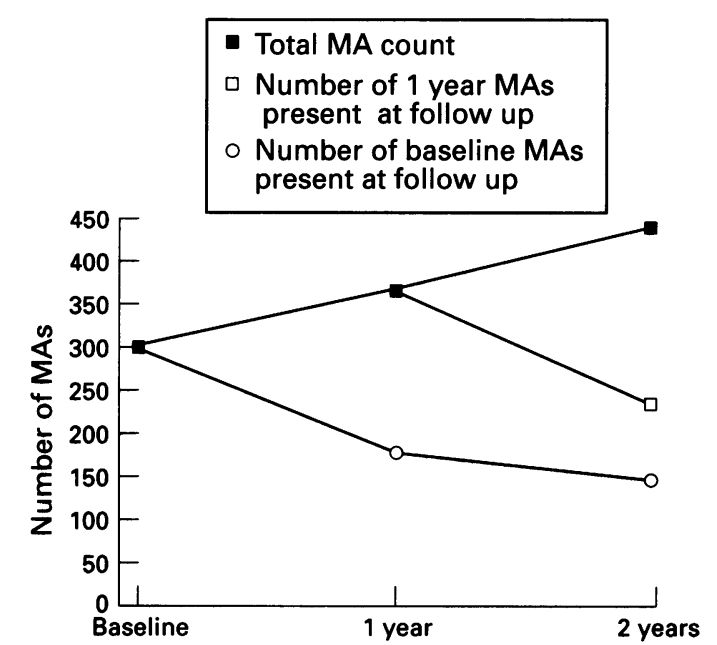

Figure 1 Pooled total microaneurysm (MA) count and disappearance of individual microaneurysms in the whole series. Note the biphasic disappearance pattern, with a faster disappearance rate during the first year of follow up compared with the second year.

lesions disappearing at the 1 year follow up but reappearing at 2 years were excluded.

\section{Results}

At the baseline the mean number of microaneurysms in the area to be analysed was 6.9 (SD $5 \cdot 6$ ), after 1 year $8 \cdot 4(7 \cdot 3)$, and after 2 years $10 \cdot 1(9 \cdot 0)$

Of the total of 298 microaneurysms found in the whole material at baseline, $174(58 \%)$ were present at the 1 year follow up and $142(48 \%)$ at the 2 year follow up (Fig 1). Thus, of the 298 microaneurysms present at baseline, 124 $(42 \%)$ were not found at the 1 year follow up and $156(52 \%)$ were not present at the 2 year follow up, but 189 new microaneurysms were present at the 1 year follow up and 294 at the 2 year follow up (Fig 2). An example of microaneurysms disappearing and forming is shown in Figure 3.

Of the total of 298 microaneurysms at baseline, 26 were not found at the 1 year follow up, although present at the 2 year follow up. This corresponds to a mean of $7 \%(12 \%)$ of the baseline microaneurysms in the individual angiograms.

When data from the identification and

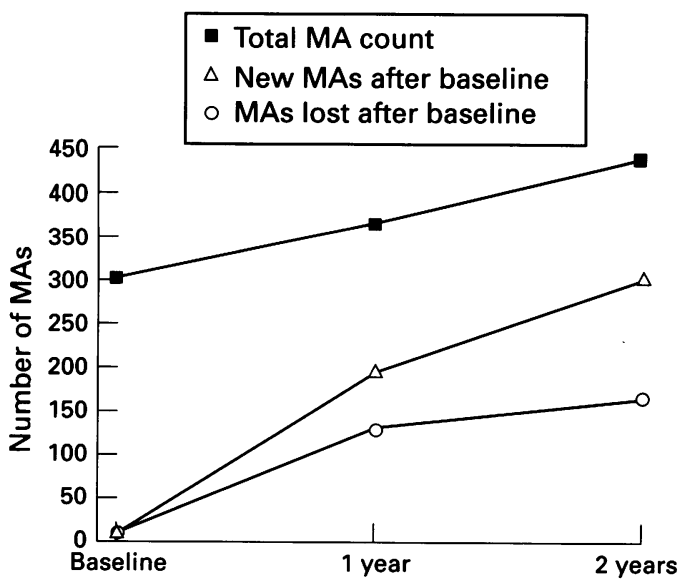

Figure 2 Pooled total microaneurysm (MA) count and cumulative disappearance and formation of microaneurysms in the whole series during a 2 year follow up. 


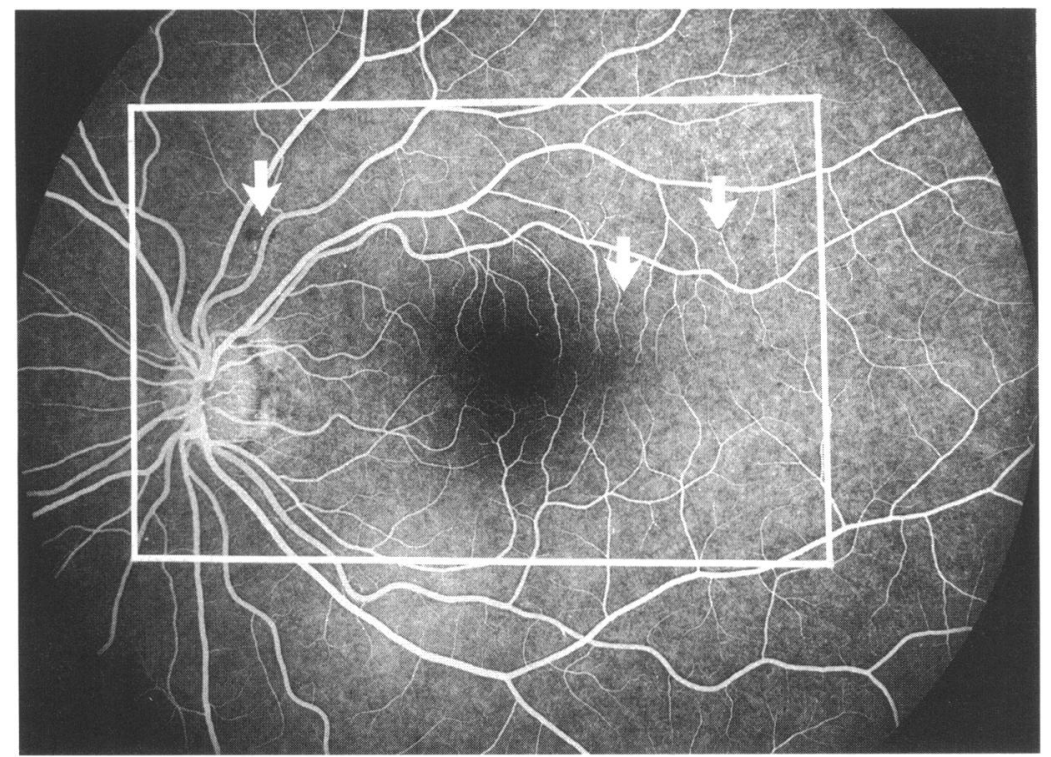

Fig $3 A$

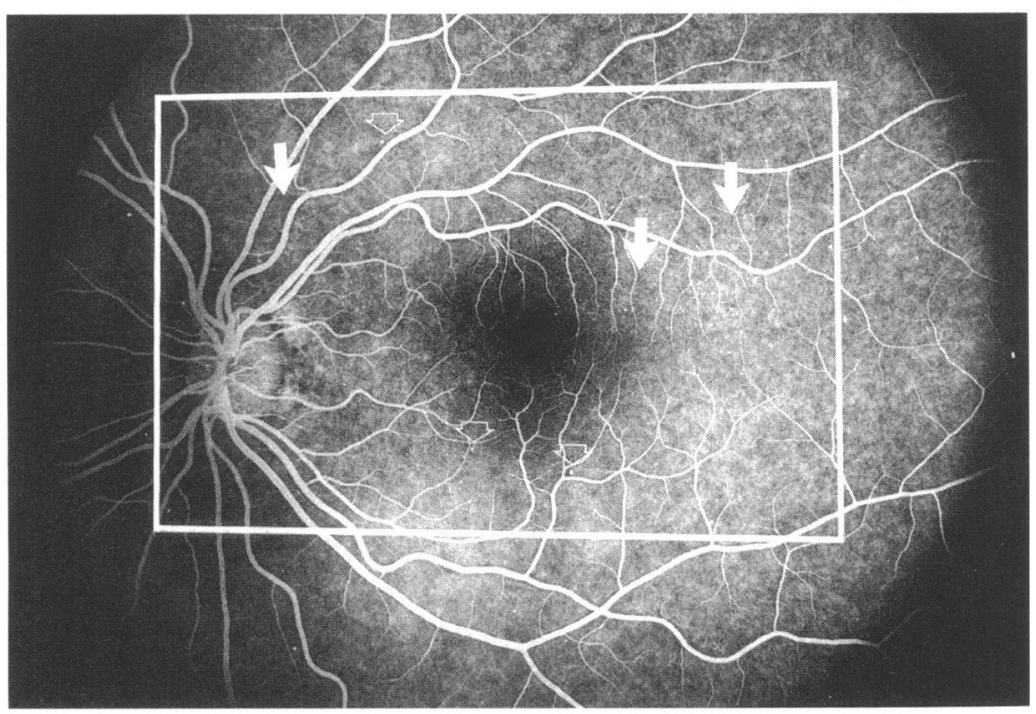

Fig $3 B$

Figure 3 Baseline $(A)$ and 2 year (B) fluoroscein angiograms from the same patient. The rectangle shows the fundus area analysed. Three microaneurysms disappeared during follow up (white arrows). Compared with baseline, there are new microaneurysms, some of them indicated by open arrows. significantly from that obtained for the same patients with the aid of a map of the baseline lesions $(p=0.4169)$.

There was a $67 \%$ agreement with the first observer $(\mathrm{TH})$ in identification of the same lesions as microaneurysms, when the same angiograms were analysed by another observer (II). The disappearance rate obtained by the second observer in a subset of patients between the baseline and 2 year angiograms was $46 \%$ $(7 \%)$, which did not differ significantly from that obtained by the first observer $(p=0 \cdot 8008)$.

When only the lesions identified by both observers were used, the disappearance rate between the baseline and 2 year angiograms was $54 \%(25 \%)$. This does not differ significantly from the value obtained by the first observer $(p=0.4188)$.

It appeared that microaneurysm count change and formation rate indexes were lower in patients with a duration of diabetes $\geqslant 19$ years. In patients with good glucose control $\left(\mathrm{HbA}_{1 \mathrm{c}} \leqslant 7.5 \mathrm{mmol}\right)$ the microaneurysm formation rate, microaneurysm count change indexes, and cumulative number of new microaneurysms were decreased with borderline statistical significance. Microaneurysm disappearance rate did not clearly correlate with these variables (Table 1 ).

\section{Discussion}

Formation of microaneurysms is an important step in the pathogenesis of diabetic retinopathy. Microaneurysm counts can be used as an indicator of progression of diabetic retinopathy and also to predict further progression of diabetic retinopathy. ${ }^{3}$ In spite of the significance of microaneurysm formation, the mechanisms involved are not completely known.

Microaneurysm formation is preceded by thickening of the capillary endothelial basement membrane, ${ }^{7-9}$ loss of pericytes, ${ }^{1011}$ and proliferation of endothelial cells, ${ }^{89}$ changes probably related to hyperglycaemia. The tendency for microaneurysms to be obliterated is probably regulated by the changes in the rheological and fibrinolytic properties of the blood in diabetics. ${ }^{12-14}$ Thus, the number of microaneurysms present and their appearance and disappearance reflect many of the systemic factors known to be altered in diabetic retinopathy.

Microaneurysm disappearance rates based on identification of individual microaneurysms have been reported previously in 1970 by Kohner et al. ${ }^{5}$ These workers found a disappearance rate of $40-80 \%$ per year, a value somewhat higher than ours. In their series, retinopathy was more severe, and a significant percentage of their patients had undergone pituitary ablation and thus differed from our patients endocrinologically. Moreover, they did not use a computerised method that would have enabled them to analyse only those lesions that were found in both the identification and verification sessions.

In principle, there are several mechanisms by which microaneurysms may not be visible in map of the baseline microaneurysms on the digitising table, the disappearance rate between the baseline and 2 year angiograms was $57 \%(23 \%)$. This value did not differ 
Table 1 Microaneurysm count change indexes and cumulative microaneurysm formation and disappearance indexes and the cumulative new microaneurysms during follow up in different groups of $H b A_{10}$ duration of diabetes, ages of the patients, and baseline microaneurysm counts. The indexes are given as percentage of the baseline microaneurysm count. Forty three eyes of 24 patients were studied. $p$ Values were calculated using the Mann-Whitney test. The values are calculated from mean value of both eyes or from the only eye in patients with data available from one eye only.

\begin{tabular}{|c|c|c|c|c|}
\hline & $\begin{array}{l}\text { Microaneurysm count } \\
\text { change index } \\
\text { Mean (SD) }\end{array}$ & $\begin{array}{l}\text { Microaneurysm formation } \\
\text { rate index } \\
\text { Mean }(S D)\end{array}$ & $\begin{array}{l}\text { Cumulative new microaneurysms } \\
\text { during follow up } \\
\text { Mean (SD) }\end{array}$ & $\begin{array}{l}\text { Microaneurysm } \\
\text { disappearance index } \\
\text { Mean (SD) }\end{array}$ \\
\hline \multicolumn{5}{|l|}{ Duration of diabetes } \\
\hline $\begin{array}{l}<19 \text { Years }(n=12) \\
\geqslant 19 \text { Years }(n=12)\end{array}$ & $\begin{array}{l}191.2(49.8) \\
129.9(72.5) \\
\mathrm{p}=0.038\end{array}$ & $\begin{array}{l}203 \cdot 0(97 \cdot 4) \\
114 \cdot 2(86 \cdot 2) \\
p=0 \cdot 015\end{array}$ & $\begin{aligned} 7 \cdot 5(7 \cdot 0) \\
8 \cdot 9(5 \cdot 9) \\
\mathrm{p}=0 \cdot 453\end{aligned}$ & $\begin{array}{l}129 \cdot 1(127 \cdot 3) \\
82 \cdot 2(31 \cdot 1) \\
\mathrm{p}=0 \cdot 273\end{array}$ \\
\hline \multicolumn{5}{|l|}{ Age } \\
\hline $\begin{array}{l}<39 \text { Years }(n=12) \\
\geqslant 39 \text { Years }(n=12)\end{array}$ & $\begin{array}{l}147.6(66.3) \\
173.5(70.9) \\
p=0.326\end{array}$ & $\begin{array}{l}152 \cdot 7(112 \cdot 5) \\
164 \cdot 6(92 \cdot 2) \\
p=0 \cdot 624\end{array}$ & $\begin{array}{l}8 \cdot 1(5 \cdot 8) \\
8 \cdot 4(7 \cdot 2) \\
p=0.863\end{array}$ & $\begin{array}{l}122 \cdot 3(124 \cdot 4) \\
88 \cdot 9(47 \cdot 6) \\
\mathrm{p}=0.686\end{array}$ \\
\hline $\begin{array}{l}\mathrm{HbA}_{1 \mathrm{c}} \\
\quad \leqslant 7 \cdot 5 \%(\mathrm{n}=6) \\
>7.5 \%(\mathrm{n}=18)\end{array}$ & $\begin{array}{l}118.7(46.9) \\
174.5(69.7) \\
\mathrm{p}=0.077\end{array}$ & $\begin{array}{l}103 \cdot 2(46 \cdot 5) \\
177 \cdot 1(107 \cdot 9) \\
p=0 \cdot 077\end{array}$ & $\begin{array}{r}4 \cdot 3(3 \cdot 7) \\
9 \cdot 5(6 \cdot 6) \\
p=0.057\end{array}$ & $\begin{array}{l}80 \cdot 3(38 \cdot 0) \\
114 \cdot 1(105 \cdot 6) \\
p=0.549\end{array}$ \\
\hline \multicolumn{5}{|c|}{ Microaneurysm at baseline } \\
\hline $\begin{array}{l}0-5(n=12) \\
6-25(n=12)\end{array}$ & $\begin{array}{l}167.9(73.5) \\
153.3(65.3) \\
p=0.564\end{array}$ & $\begin{array}{l}188 \cdot 5(116 \cdot 1) \\
128 \cdot 7(76 \cdot 0) \\
p=0 \cdot 184\end{array}$ & $\begin{array}{l}3 \cdot 6(2 \cdot 4) \\
12 \cdot 6(6 \cdot 2) \\
\mathrm{p}=0 \cdot 0001\end{array}$ & $\begin{array}{l}135 \cdot 3(122 \cdot 9) \\
76 \cdot 0(36 \cdot 4) \\
\mathrm{p}=0 \cdot 106\end{array}$ \\
\hline
\end{tabular}

fluorescein angiograms. Occlusion of a capillary naturally leads to disappearance of the microaneurysm within the capillary. A thrombus within the microaneurysm may also result in its disappearance, and so may temporary adherence of platelets or leucocytes to the microaneurysm walls. ${ }^{12}$

The analysis of microaneurysms from fluorescein angiograms or colour photographs of the fundus is subject to errors, which include dissimilarities in photography and in interpretation of the lesions found. In spite of these inaccuracies, the study of fundus images has proved to be a reproducible means of staging diabetic retinopathy.

Our method for localising and identifying microaneurysms is naturally subject to the same potential errors. Marking the lesions found in the previous image on the paper in which the angiogram is projected might lead to increased detection of old lesions and thus to underestimation of the disappearance rate. However, in this material the disappearance rate was closely similar when the lesions were not marked. Further, the intra-analyser and inter-analyser variations were relatively small. Although we combined data from two independent microaneurysm localising sessions, the results would have been essentially the same if data from only one of the sessions had been used.

We used $60^{\circ}$ angiograms and analysed fluorescein angiograms at only one phase of fluorescein transit. It is possible that some of the smaller microaneurysms visible in $30^{\circ}$ images may not have been recorded in our material. The use of only transit phase fluorescein angiograms probably visualised only microaneurysms that were clearly open. Microaneurysms partially filled by a thrombus staining only at late phase of the angiogram may not have been found in our analysis. These conditions apply similarly to baseline and follow up angiograms and are thus unlikely to affect the disappearance and formation rates obtained.

We analysed only the central area of the retina. Thus, the microaneurysm counts are not comparable with those obtained from the seven standard fields of the Airlie House classification. The disappearance and formation rates may also be different in more peripheral areas of the retina. As we analysed fluorescein angiograms only, we cannot exclude the possibility that some retinal pigment epithelium defects may have been classified as microaneurysms. It seems very unlikely, however, that such lesions would have undergone disappearance and formation at the rates observed in this study.

In our study, we found that only $40 \%$ to $50 \%$ of baseline microaneurysms were detectable after 2 years. There seemed to be a higher disappearance rate of baseline microaneurysms from baseline to year 1 than during the interval from year 1 to year 2 . This suggests that there may be two populations of microaneurysms, one with a shorter half life than the other. A similar pattern of microaneurysm disappearance was also detected by Kohner et al. ${ }^{5}$ A proportion of microaneurysms was also detected only at the 1 year follow up. It is thus possible that the lifespan of these microaneurysms is less than 2 years.

About $7 \cdot 3 \%$ of the microaneurysms were present at the baseline and had disappeared at the 1 year follow up, but were detected again at the 2 year follow up. This variability may have been due to changes in the quality of the angiograms, but it is also possible that some of the microaneurysms are occluded temporarily by fibrin or platelet thrombi and then recanalised, so reappearing in the 2 year fluorescein angiogram.

In most patients, the loss of microaneurysms was more than compensated for by the formation of new microaneurysms, there being a small net increase in the microaneurysm count. These results indicate that changes in microaneurysm counts reflect the balance between the formation and disappearance of microaneurysms. An increase in microaneurysm count, commonly used to measure worsening of early retinopathy, may thus result from increased formation or decreased disappearance of microaneurysms. When individual microaneurysms are followed up, these two phenomena can be dissociated to yield the disappearance rate, as well as the rate of formation of new microaneurysms. 
In patients with diabetes of long duration without proliferative retinopathy, the background retinopathy is attenuated, as reflected by a somewhat lower microaneurysm formation rate in patients with the duration of diabetes 19 or more years.

The cumulative number of new microaneurysms was somewhat elevated in patients with $\mathrm{HbA}_{1 \mathrm{c}}>7.5 \mathrm{mmol}$; microaneurysm formation rate and microaneurysm count change indexes also showed a trend of being elevated in these patients. This suggests that the microaneurysm formation rate and microaneurysm count change both respond to hyperglycaemia.

A more definite assessment of the differences between these variables is not possible in this fairly homogeneous material with relatively stable background retinopathy during follow up. To evaluate the possible differences in the sensitivity of these variables in responding to hyperglycaemia, larger materials with clearly different glucose control are needed.

The microaneurysm disappearance rate did not differ significantly between the different groups of $\mathrm{HbA}_{1 \mathrm{c}}$, or with the duration of diabetes, or other clinical or demographic variables.

It is thus possible that the microaneurysm disappearance rate is not directly connected with glycaemic control. We did not measure serum fibrinogen or plasminogen activator inhibitor levels. ${ }^{15-18}$ Whether the microaneurysm disappearance rate is connected with these factors potentially favouring thrombus formation remains to be evaluated in further studies.

Our results highlight the fact that background retinopathy is not merely a process of cumulative increase in the number of microaneurysms, but that the microaneurysm count reflects a dynamic balance between microaneurysm formation and disappearance. It is possible that, with follow up of individual microaneurysms, more information on the progression patterns of early diabetic retinopathy can be obtained.

Supported by grants from The Finnish Eye Foundation (Silmäsäätiö), Helsinki, Finland, the Friends of the Blind Foundation, Helsinki, Finland, and the Science Foundation of Orion Pharmaceuticals Ltd, Finland.

1 Diabetic Retinopathy Study Research Group. A modification of the Airlie House classification of diabetic retinopathy. The seventh report from the Diabetic Retinopathy opathy. The seventh report from the Diabetic Retino

2 Grading Diabetic Retinopathy from Stereoscopic Color Fundus Photographs - An extension of the modified Airlie House classification. ETDRS report number 10. Ophthalmology 1991; 98: 786-806.

3 Klein R, Meuer SM, Moss SE, Klein BE. The relationship of retinal microaneurysm counts to the 4 year progression of diabetic retinopathy. Arch Ophthalmol 1989; 107: 1780-5.

4 Kohner EM, Sleightholm M. Does microaneurysm count reflect severity of early diabetic retinopathy? reflect severity of early

5 Kohner EM, Dollery CT. The rate of formation and disappearance of microaneurysms in diabetic retinopathy. Eur $\mathcal{F}$ Clin Invest 1970; 1: 167-71.

6 Hellstedt T, Palsi V-P, Immonen I. A computerized system for localization of diabetic lesions from fundus images. Acta Ophthalmol (Copenh) 1994; 72: 352-6.

7 Ashton N. Vascular basement membrane changes in diabetic retinopathy. Montgomery lecture, 1973. $\mathrm{Br} \mathcal{F}$ Ophthalmol 1974; 58: 344-7.

8 Frank $\mathrm{RN}$. On the pathogenesis of diabetic retinopathy. Ophthalmology 1984; 91: 626-34.

9 Frank RN. On the pathogenesis of diabetic retinopathy. A 1990 update. Ophthalmology 1991; 98: 586-93

10 Speiser P, Gittelsohn AM, Patz A. Studies on diabetic retinopathy. III. Influence of diabetes on intramural pericytes. Arch Ophthalmol 1968; 80: 332-7.

$11 \mathrm{De}$ Oliveira F. Pericytes in diabetic retinopathy. $\mathrm{Br} F$ Ophthalmol 1966; 50: 134-43.

12 Little HL. Alterations in blood elements in the pathogenesis of diabetic retinopathy. Ophthalmology $1981 ; 88$ : 647-54.

13 Porta M, Hilgard P, Kohner EM Platelet shape change abnormalities in diabetic retinopathy. Diabetologia 1980; 18: $217-21$

14 Zatz R, Brenner BM. Pathogenesis of diabetic microangiopathy. The hemodynamic view. Am $\mathcal{F}$ Med 1986; 80:

15 Kruithof EKO, Tran-Thang C, Bachmann F. The fastacting inhibitor of tissuetype plasminogen activator in plasma is also the primary plasma inhibitor of urokinase. Thromb Haemost 1986; 55: 65-9.

16 Kruithof EKO. Plasminogen activator inhibitors - a review. Enzyme 1988; 40: 113-21.

17 Vague P, JuhanVague I, Aillaud MF, Badier C, Viard R, Alessi MC, et al. Correlation between blood fibrinolytic activity, plasminogen activator inhibitor level, plasma insulin level and relative body weight in normal and obese insulin level and relative body weight in
subjects. Metabolism 1986; 35: 250-3.

18 Auwerx J, Bouillon R, Collen D, Gebores T. Tissue-type plasminogen activator antigen and plasminogen activator inhibitor in diabetes mellitus. Arteriosclerosis 1988; 8: 68-72. 